[11] A. Brambilla and D. D'Amore, "Energy-based control of numerical errors in time-domain simulation of dynamic circuits," IEEE Trans. Circuits Syst., to be published.

[12] A. Brambilla, D. D'Amore, and E. Dallago, "A new numerical method for steady-state circuit analysis," in Proc. 11th Eur. Conf. Circuit Theory and Design, Davos, Switzerland, Aug. 30-Sept. 3, 1993, pp. 1671-1676.

[13] A. Brambilla, D. D'Amore, and M. Santomauro, "Simulation of autonomous circuits in the time domain," in Proc. ECCTD'95, Istanbul, Turkey, Aug. 28-Sept. 1, 1995, pp. 399-402.

[14] R. Telichevesky, K. Kundert, I. Elfadel, and J. White, "Fast simulation algorithms for RF circuits," in Proc. CICC'96, May 1996, pp. 437-444.

[15] K. Kundert, "Simulation methods for RF integrated circuits," in Proc. ICCAD'97, Nov. 1997, pp. 1-14

[16] H. Jokinen, "Computation of the steady-state solution of nonlinear circuits with time-domain and large-signal-small-signal analysis methods," Acta Polytech. Scand., Electr. Eng. Ser., vol. 87, 1997.

\section{A Robust Tracking Control for Chaotic Chua's Circuits via Fuzzy Approach}

\author{
Yeong-Chan Chang
}

\begin{abstract}
This paper addresses the problem of designing robust tracking controls for nonlinear chaotic Chua's circuits involving plant uncertainties and external disturbances. A hybrid adaptive-robust tracking control scheme which is based upon a combination of the $H^{\infty}$ tracking theory, variable structure system (VSS) control algorithm, and adaptive fuzzy control is developed such that all the states and signals of the closed-loop system are bounded and an $H^{\infty}$ tracking control from the tracking error to the external disturbance is guaranteed. In contrast to the previous investigations of controlling Chua's circuits the controller developed here can be extended to handle a broader class of uncertain nonlinear chaotic Chua's circuits.
\end{abstract}

Index Terms-Chaotic Chua's circuits, $H^{\infty}$ tracking control, hybrid adaptive-robust design, Riccati-like matrix equation.

\section{INTRODUCTION}

The control of nonlinear chaotic Chua's circuits is an important topic for numerous practical applications since this circuit exhibits a wide variety of nonlinear dynamic phenomena such as bifurcations and chaos [5], [6], [18]. This chaotic circuit possesses the property of simplicity and universality, and has become a standard prototype for investigation of chaos. A significant research attention has been paid toward studying the control of such circuits in the past few years [2]-[6], [11], [14], [15], [17], [18]. In most of these previous investigations, the plant dynamics is assumed to be available for implementation or external disturbances are neglected. However, in practical applications, plant uncertainties and external disturbances which may affect the tracking performance are inevitable. Therefore, development of an alternative control approach to efficiently treat the robust tracking control of chaotic Chua's circuits involving a large class of uncertainties and variations is highly desirable.

Fuzzy control has recently found extensive applications for a wide variety of industrial systems and many adaptive fuzzy control

Manuscript received May 10, 2000; revised February 15, 2001. This paper was recommended by Associate Editor T. Saito.

The author is with the Department of Electrical Engineering, Kun-Shan University of Technology, Yung-Kang, Tainan Hsien, Taiwan, R.O.C. (e-mail: ycchang@mail.ksut.edu.tw).

Publisher Item Identifier S 1057-7122(01)05393-4.

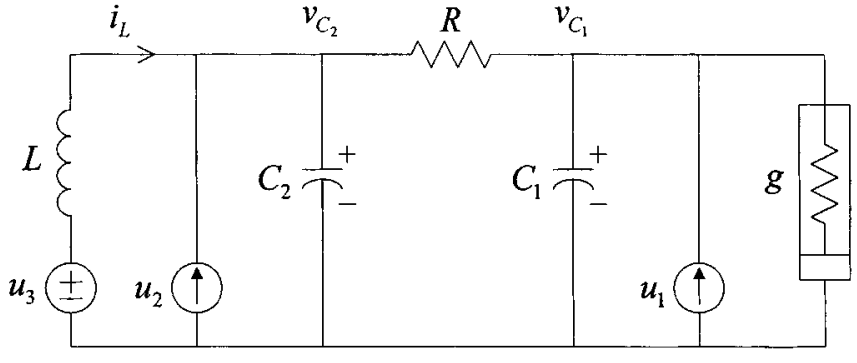

Fig. 1. Diagram of nonlinear chaotic Chua's circuit.

schemes have been developed [3], [7], [12], [13], [16]. Specially, some fuzzy-based control schemes are also developed to treat the control of chaotic circuits [2], [5], [15]. In [2] a simple fuzzy logic based intelligent mechanism was developed for predicting and controlling an uncertain chaotic system to a desired target. Chen and Dong [5] applied fuzzy inference systems to identify and control chaotic systems. Tanaka et al. [15] developed a unified approach to controlling chaos via an linear matrix inequality (LMI)-based fuzzy control system design.

This paper addresses the robust tracking control for a large class of nonlinear chaotic Chua's circuits in the presence of plant uncertainties and external disturbances. Nonlinear $H^{\infty}$ tracking theory, variable structure system (VSS) control algorithm and fuzzy control design are combined together to construct a VSS indirect adaptive fuzzybased $H^{\infty}$ tracking controller such that the resulting closed-loop circuit system guarantees a satisfactorily transient and asymptotic performance in the sense that the tracking error can be made as small as possible in terms of $L_{\infty}$ boundedness and $H^{\infty}$ tracking performance. In order to implement the developed controller, knowledge of the dynamic model of nonlinear Chua's circuits doesn't require for feedback and moreover only a linear Riccati-like equation must be solved. Consequently, the control scheme developed in this study can be easily implemented from the viewpoint of practical applications.

In Section II, the tracking control problem of nonlinear chaotic Chua's circuits is presented. Section III develops a hybrid adaptive-robust fuzzy-based controller. Section IV presents a simulation example. Finally, the conclusion is given in Section V.

\section{PRELiminary}

\section{A. Problem Statement}

The chaotic Chua's circuit, as shown in Fig. 1, is a simple electronic system that consists of one inductor $L$, two capacitors $C_{1}, C_{2}$, one linear resistor $R$, and one nonlinear resistor $g$. The dynamic equations of Chua's circuit with control inputs are described by [5], [18]

$$
\begin{aligned}
\dot{v}_{c_{1}} & =\frac{1}{C_{1}}\left(\frac{1}{R}\left(v_{c_{2}}-v_{c_{1}}\right)-g\left(v_{c_{1}}\right)+u_{1}\right)+d_{1} \\
\dot{v}_{c_{2}} & =\frac{1}{C_{2}}\left(\frac{1}{R}\left(v_{c_{1}}-v_{c_{2}}\right)+i_{L}+u_{2}\right)+d_{2} \\
\dot{i}_{L} & =\frac{1}{L}\left(-v_{c_{2}}+u_{3}\right)+d_{3}
\end{aligned}
$$

where

$$
\begin{array}{ll}
i_{L} & \text { current through the inductor } L ; \\
v_{c_{1}}, v_{c_{2}} & \text { voltages across } C_{1} \text { and } C_{2}, \text { respectively; } \\
g(\cdot) & \text { current through the nonlinear resistor; } \\
u_{1}, u_{2}, u_{3} & \text { control inputs; } \\
d_{1}, d_{2} \text { and } d_{3} & \text { external disturbances. } \\
\text { Let } x(t) \triangleq\left[v_{c_{1}}(t), v_{c_{2}}(t), i_{L}(t)\right]^{T} \text { be the state variable. }
\end{array}
$$


Give a desired reference signal $x_{d}(t) \triangleq\left[x_{d_{1}}(t), x_{d_{2}}(t), x_{d_{3}}(t)\right]^{T}$ which is assumed to be bounded and continuously differentiable, i.e., there is a compact set $\Omega_{d}$ such that $x_{d}(t) \in \Omega_{d}$ for all $t \geq 0$. The objective of this paper is to design an adaptive-robust tracking controller for nonlinear chaotic Chua's circuits (1)-(3) involving plant uncertainties and external disturbances such that the resulting closed-loop system guarantees that all the states and signals are bounded and the tracking error should be as small as possible.

Taking $e(t) \triangleq x(t)-x_{d}(t)=\left[v_{c_{1}}(t)-x_{d_{1}}(t), v_{c_{2}}(t)-\right.$ $\left.x_{d_{2}}(t), i_{L}(t)-x_{d_{3}}(t)\right]^{T}$, the tracking error dynamic equation can be expressed as

$$
\dot{e}=F(x)+G(x) u+d-\dot{x}_{d}
$$

where $u \triangleq\left[u_{1}, u_{2}, u_{3}\right]^{T}, d \triangleq\left[d_{1}, d_{2}, d_{3}\right]^{T}$, and

$$
\begin{aligned}
& F(x) \triangleq\left[\begin{array}{l}
f_{1}(x) \\
f_{2}(x) \\
f_{3}(x)
\end{array}\right] \triangleq\left[\begin{array}{c}
\frac{1}{C_{1}}\left(\frac{1}{R}\left(v_{c_{2}}-v_{c_{1}}\right)-g\left(v_{c_{1}}\right)\right) \\
\frac{1}{C_{2}}\left(\frac{1}{R}\left(v_{c_{1}}-v_{c_{2}}\right)+i_{L}\right) \\
\frac{1}{L}\left(-v_{c_{2}}\right)
\end{array}\right] \\
& G(x) \triangleq\left[\begin{array}{ccc}
\frac{1}{C_{1}} & 0 & 0 \\
0 & \frac{1}{C_{2}} & 0 \\
0 & 0 & \frac{1}{L}
\end{array}\right] .
\end{aligned}
$$

It is clear that if the function $F(x)$ and the matrix $G(x)$ are well-known, by employing the technique of feedback linearization [9] there exists a suitable control law such that the resulting closed-loop system can be shown to achieve a satisfactorily tracking performance. However, in practical circuit systems, the inductor, capacitor, and resistor may have uncertain variations around their nominal values due to heating. The parameters and characteristics of electronic elements may vary while the circuit system has operated for a long time, and the circuit system may receive unpredictable interference from the environment where it resides, etc. Therefore, in order to efficiently control the chaotic Chua's circuit it is necessary to consider the effects due to plant uncertainties and external disturbances.

In this study, the values of the inductor $L$, capacitors $C_{1}$ and $C_{2}$, and resistors $R$ and $g(\cdot)$ are assumed to be unknown. Therefore, the nonlinear dynamics $F(x)$ and $G(x)$ are also unknown and cannot be available directly in the robust control design. The philosophy of our tracking design is expected that the fuzzy approximator equipped with an adaptive algorithm is introduced first to learn the uncertain dynamics. Next, two additional robust control algorithms, i.e., VSS algorithm and nonlinear $H^{\infty}$ control algorithm, are employed to efficiently attenuate the effects on the tracking error due to the fuzzy approximation error and the external disturbance.

Two cases with respect to different knowledge of the input gain matrix will be proposed, sequentially. One is assumed that the values of $C_{1}, C_{2}$ and $L$ can be split into a nominal part plus an uncertain part and the other is assumed that these three values are nonlinear time-varying and unknown.

\section{B. Description of Fuzzy Systems}

The basic configuration of the fuzzy system shown in Fig. 2 is constructed from the fuzzy If-Then rules using some specific

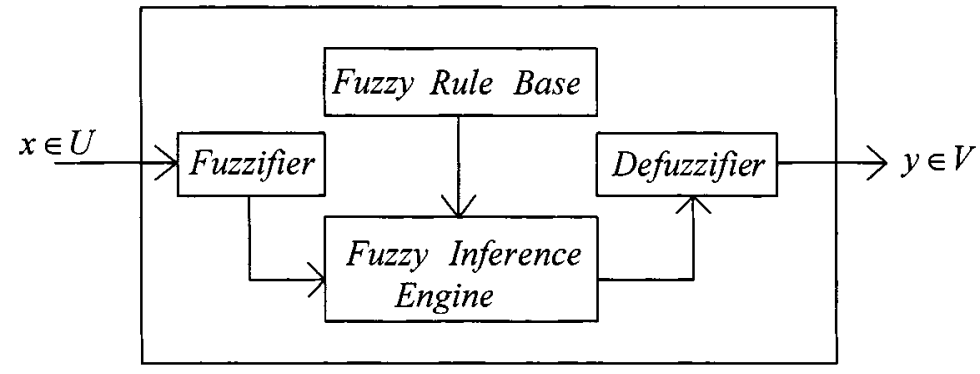

Fig. 2. The basic configuration of a fuzzy logic system.

inference, fuzzification, and defuzzification strategies [13], [16]. The fuzzy system performs a mapping from $U \subset R^{N}$ to $V \subset R$. The fuzzifier maps a crisp point in $U$ into a fuzzy set in $U$. The fuzzy rule base consists of a collection of fuzzy If-Then rules such as

$$
\begin{aligned}
& R^{(l)}: \text { If } x_{1} \text { is } F_{1}^{l}, \cdots, x_{N} \text { is } F_{N}^{l}, \\
& \text { Then } y \text { is } G^{l}
\end{aligned}
$$

in which $x=\left(x_{1}, \ldots, x_{N}\right)^{T} \in U$ and $y \in V \subset R$ are the input and output of the fuzzy system, respectively, $F_{i}^{l}, i=1,2, \ldots, N$ and $G^{l}$ are fuzzy sets, and $l=1, \ldots, M$ where $M$ denotes the number of fuzzy If-Then rules. The fuzzy inference engine performs a mapping from fuzzy sets in $U$ to fuzzy sets in $V$, based upon the fuzzy If-Then rules and the compositional rule of inference. The defuzzifier maps a fuzzy set in $V$ to a crisp point in $V$.

According to the universal approximation theorem [13], [16] the fuzzy system with center-average defuzzifier, product inference and singleton fuzzifier is of the following form:

$$
y(x)=\frac{\sum_{l=1}^{M} \theta_{l}\left(\prod_{i=1}^{N} \mu_{F_{i}^{l}}\left(x_{i}\right)\right)}{\sum_{l=1}^{M}\left(\prod_{i=1}^{N} \mu_{F_{i}^{l}}\left(x_{i}\right)\right)}
$$

where $\mu_{F^{l}}(\cdot)$ is the numbership function of the fuzzy set $F_{i}^{l}$ and $\theta_{l}$ is the point at which $\mu_{G^{l}}$ achieves its maximum value. Moreover, for any given real continuous function $f(x)$ on a compact subset $U \subset R^{N}$ and arbitrary $\epsilon>0$, there exists a fuzzy system $y(x)$ in the form of (6) such that $\max _{x \in U}\|f(x)-y(x)\|<\epsilon$.

\section{Controller Design AND Stability Analysis}

First, we shall focus on the case that $L, C_{1}$ and $C_{2}$ can be partitioned as $L=L_{0}+\Delta L, C_{1}=C_{10}+\Delta C_{1}$ and $C_{2}=C_{20}+\Delta C_{2}$ where $L_{0}, C_{10}$ and $C_{20}$ denote the known nominal values and $\Delta L, \Delta C_{1}$ and $\Delta C_{2}$ denote the unknown nonlinear time-varying perturbations. After some simple manipulations, the input matrix $G(t, x)$ can be expressed as $G(t, x)=$ $G_{0}+\Delta G(t, x)$ where $G_{0}=\operatorname{diag}\left[1 / C_{10}, 1 / C_{20}, 1 / L_{0}\right]$ and $\Delta G=\operatorname{diag}\left[-\left(\Delta C_{1} / C_{10} C_{1}\right),-\left(\Delta C_{2} / C_{20} C_{2}\right),-\left(\Delta L / L_{0} L\right)\right]$.

Take the universal approximation system $\hat{F}(x, \Theta)$ with $x \in U_{x}$ for some compact set $U_{x} \subset R^{3}$, to approximate the uncertain term $F(x)$ where $\Theta$ contains the tunable approximation parameters. Let $\hat{f}_{i}\left(x, \Theta_{i}\right), i=1,2,3$ denote the $i$ th component of $\hat{F}(x, \Theta)$ and use to approximate $f_{i}(x)$. From (6) $\hat{f}_{i}\left(x, \Theta_{i}\right)$ can be expressed as

$$
\hat{f}_{i}\left(x, \Theta_{i}\right)=\zeta_{i}^{T}(x) \Theta_{i}, \quad i=1,2,3
$$


where $\Theta_{i} \triangleq\left[\theta_{i 1}, \ldots, \theta_{i m_{i}}\right]^{T} \in R^{m_{i}}$ for some $m_{i}>0$, is a parameter vector and $\zeta_{i}(x) \triangleq\left[\zeta_{i 1}(x), \ldots, \zeta_{i m_{i}}(x)\right]^{T}$ is a regressive vector with the regressor $\zeta_{i l}(x)$ defined as

$$
\zeta_{i l}(x)=\frac{\prod_{j=1}^{3} \mu_{F_{j}^{l}}\left(x_{j}\right)}{\sum_{l=1}^{m_{i}}\left(\prod_{j=1}^{3} \mu_{F_{j}^{l}}\left(x_{j}\right)\right)}, \quad l=1, \ldots, m_{i} .
$$

Here, the linearly parametrized fuzzy model [3], [12], [13], [16] is employed in the approximation procedure. That is, the membership functions $\mu_{F_{j}^{l}}\left(x_{j}\right)$ for $1 \leq l \leq m_{i}$ and $1 \leq j \leq 3$ are specified beforehand and the number $m_{i}$ is given so that $\hat{f}_{i}\left(x, \Theta_{i}\right)$ can approximate $f_{i}(x)$ as best as possible. Consequently, the fuzzy system $\hat{F}(x, \Theta)$ can be expressed as

$$
\hat{F}(x, \Theta)=\left[\begin{array}{ccc}
\zeta_{1}^{T}(x) & 0 & 0 \\
0 & \zeta_{2}^{T}(x) & 0 \\
0 & 0 & \zeta_{3}^{T}(x)
\end{array}\right]\left[\begin{array}{c}
\Theta_{1} \\
\Theta_{2} \\
\Theta_{3}
\end{array}\right] \triangleq Y(x) \Theta
$$

where $Y(x) \in R^{3 \times m}$ is a basis matrix and $\Theta \triangleq\left[\Theta_{1}^{T}, \Theta_{2}^{T}, \Theta_{3}^{T}\right]^{T} \in$ $R^{m}$ with $m=m_{1}+m_{2}+m_{3}$.

According to the universal approximation theorem, there exists an optimal approximation parameter $\Theta^{*}$ [3], [13], [16] such that $\hat{F}\left(x, \Theta^{*}\right)$ can approximate $F(x)$ as best as possible. As in many previous adaptive fuzzy designs, $\Theta^{*}$ will be learned by using an adaptive algorithm. Suppose the constrained region of $\Theta$ is chosen to be a convex hypercube. That is, consider $\Omega_{0} \triangleq\left\{\Theta \mid b_{i j} \leq \theta_{i j} \leq c_{i j}, 1 \leq j \leq m_{i}, 1 \leq i \leq 3\right\}$ and $\Omega_{\theta} \triangleq\left\{\Theta \mid b_{i j}-\delta \leq \theta_{i j} \leq c_{i j}+\delta, 1 \leq j \leq m_{i}, 1 \leq i \leq 3\right\}$ where the values of $b_{i j}, c_{i j}$ and $\delta>0$ can be arbitrarily specified by the designer. Give a smooth function $\Phi \triangleq\left[\Phi_{1}^{T}, \Phi_{2}^{T}, \Phi_{3}^{T}\right]^{T}$ where $\Phi_{1} \in R^{m_{1} \times 1}, \Phi_{2} \in R^{m_{2} \times 1}$ and $\Phi_{3} \in R^{m_{3} \times 1}$ should be specified later. Therefore, the smooth projection algorithm with respect to $\Omega_{\theta}$ can be expressed as [10]

$\operatorname{Proj}[\Phi, \Theta] \triangleq \begin{cases}\bar{\phi}_{i j}, & \text { if }\left(\theta_{i j}>c_{i j} \text { and } \phi_{i j}>0\right) \\ \check{\phi}_{i j}, & \text { if }\left(\theta_{i j}<b_{i j} \text { and } \phi_{i j}<0\right) \\ \phi_{i j}, & \text { otherwise, } 1 \leq j \leq m_{i}, 1 \leq i \leq 3\end{cases}$

where $\bar{\phi}_{i j} \triangleq\left(1+\left(c_{i j}-\theta_{i j} / \delta\right)\right) \phi_{i j}, \check{\phi}_{i j} \triangleq\left(1+\left(\theta_{i j}-b_{i j} / \delta\right)\right) \phi_{i j}$ and $\phi_{i j}$ denotes the $j$ element of $\Phi_{i}$.

Let $\Delta F(x)=F(x)-\hat{F}\left(x, \Theta^{*}\right)$ be the minimum approximation error and $\Delta f_{i}$ be the $i$ element of $\Delta F$. Throughout this section we need the following assumptions.

A1) There exists a constant $0<\kappa<1$ such that $\left|\lambda_{i}\left(\Delta G G_{0}^{-1}\right)\right| \leq \kappa, \forall 1 \leq i \leq 3$.

A2) There exists a constant $\epsilon_{f}>0$ such that $\left|\Delta f_{i}\right| \leq \epsilon_{f}, \forall$ $1 \leq i \leq 3$.

A3) There exists a constant $M_{d}>0$ such that $\int_{0}^{\infty}\|d(t)\|^{2} d t \leq$ $M_{d}$, i.e., $d \in L_{2}[0, \infty)$.

The assumption A1) is equivalent to $\left|\Delta C_{1} / C_{1}\right| \leq \kappa,\left|\Delta C_{2} / C_{2}\right| \leq$ $\kappa$ and $|\Delta L / L| \leq \kappa$, and so it always holds for small parameter perturbations. According to the universal approximation theorem the assumption A2 also holds. The assumption A3 implies the external disturbance is of finite-energy. This kind of disturbance usually appears in a physically operating circuit.

Theorem 1: Consider the nonlinear chaotic Chua's circuit (1)-(3). Suppose that Assumptions A1)-A3) are satisfied. If there exists a sym- metric positive matrix $P=P^{T}>0$ satisfying the Riccati-like equation

$$
-K^{T} P-P K+Q+P\left(\frac{1}{\rho^{2}} I_{3 \times 3}-(1-\kappa) R^{-1}\right) P=0
$$

where $\rho>0$ is a prescribed attenuation level, $Q=Q^{T}>0$ is a prescribed weighting matrix, $K \triangleq \operatorname{diag}\left[k_{1}, k_{2}, k_{3}\right]$ for some constants $k_{i}>0$ is a state feedback control gain, and $R \triangleq \operatorname{diag}\left[r_{1}, r_{2}, r_{3}\right]$ for some constants $r_{i}>0$ is an $H^{\infty}$ control gain, then the following VSS adaptive fuzzy-based control law

$$
u=G_{0}^{-1}\left(-Y(x) \Theta+\dot{x}_{d}-K e+u_{h}+u_{s}\right)
$$

with

$$
\begin{aligned}
u_{h} & =-\frac{1}{2} R^{-1} P e \\
u_{s} & =-\frac{\epsilon_{f}+\kappa M_{e}}{1-\kappa} \operatorname{sgn}(P e) \\
\dot{\Theta} & =\gamma \operatorname{Proj}[\Phi, \Theta]
\end{aligned}
$$

where $M_{e} \triangleq\left\|-Y \Theta+\dot{x}_{d}-K e\right\|, \operatorname{sgn}(P e) \triangleq\left[\operatorname{sgn}\left((P e)_{1}\right)\right.$, $\left.\operatorname{sgn}\left((P e)_{2}\right), \operatorname{sgn}\left((P e)_{3}\right)\right]^{T}, \Phi \triangleq Y^{T} P e$, and $\gamma$ denotes the adaptive gain, guarantees that i) all the variables of the closed-loop system (1)-(3), (12)-(15) are bounded and ii) an $H^{\infty}$ tracking performance from $d(t)$ to $e(t)$ is achieved, i.e.,

$$
\begin{aligned}
& \int_{0}^{T}\|e(t)\|_{Q}^{2} d t \leq 2 V(0)+\rho^{2} \int_{0}^{T}\|d(t)\|^{2} d t \\
& \forall 0 \leq T<\infty
\end{aligned}
$$

where $V(0)$ denotes the initial conditions.

Proof: Taking into account the minimum approximation error and the control law (12), the error dynamic equation (4) can be rewritten as

$$
\begin{aligned}
\dot{e} & =\hat{F}\left(x, \Theta^{*}\right)+\Delta F(x)+G_{0} u+\Delta G(t, x) u+d-\dot{x}_{d} \\
& =-K e-Y(x) \tilde{\Theta}+\Delta F(x)+\Delta G(t, x) u+u_{h}+u_{s}+d
\end{aligned}
$$

where $\tilde{\Theta}=\Theta-\Theta^{*}$. Choose a Lyapunov function as

$$
V=\frac{1}{2} e^{T} P e+\frac{1}{2 \gamma} \tilde{\Theta}^{T} \tilde{\Theta}
$$

By completing the squares and using the control input $u_{h}$ in (13), the time derivative $\dot{V}$ along the error trajectory (17) is

$$
\begin{aligned}
\dot{V}= & \frac{1}{2} e^{T}\left(-K^{T} P-P K\right) e+u_{h}^{T}\left(I+\Delta G G_{0}^{-1}\right)^{T} P e \\
& +d^{T} P e+u_{s}^{T}\left(I+\Delta G G_{0}^{-1}\right)^{T} P e \\
& +\left(\Delta F+\Delta G G_{0}^{-1}\left(-Y \Theta+\dot{x}_{d}-K e\right)\right)^{T} P e \\
& -\tilde{\Theta}^{T} Y^{T} P e+\frac{1}{\gamma} \dot{\Theta}^{T} \tilde{\Theta} \\
= & \frac{1}{2} e^{T}\left(-K^{T} P-P K\right. \\
& \left.+P\left(\frac{1}{\rho^{2}} I-R^{-1}\left(I+\Delta G G_{0}^{-1}\right)^{T}\right) P\right) e \\
& -\frac{1}{2}\left(\frac{1}{\rho} P e-\rho d\right)^{T}\left(\frac{1}{\rho} P e-\rho d\right)+\frac{1}{2} \rho^{2} d^{T} d \\
& +u_{s}^{T}\left(I+\Delta G G_{0}^{-1}\right)^{T} P e+\left(\Delta F+\Delta G G_{0}^{-1}\right. \\
& \left.\cdot\left(-Y \Theta+\dot{x}_{d}-K e\right)\right)^{T} P e-\tilde{\Theta}^{T} Y^{T} P e+\frac{1}{\gamma} \dot{\Theta}^{T} \tilde{\Theta} .
\end{aligned}
$$


From the update law (15) which is a standard smooth projection algorithm [10] we can guarantee that $1 / \gamma \dot{\Theta}^{T} \tilde{\Theta}-\tilde{\Theta}^{T} Y^{T} P e \leq 0$ and $\Theta(t) \in \Omega_{\theta}$ for all $t \geq 0$ if $\Theta(0) \in \Omega_{0}$. Moreover, by $u_{s}$ in (14) and the assumptions A1)-A2), we get

$$
\begin{aligned}
u_{s}^{T}\left(I+\Delta G G_{0}^{-1}\right)^{T} P e+\left(\Delta F+\Delta G G_{0}^{-1}\left(-Y \Theta+\dot{x}_{d}-K e\right)\right)^{T} P e \\
\leq-\left(\epsilon_{f}+\kappa M_{e}\right) \sum_{i=1}^{3}\left|(P e)_{i}\right|+\sum_{i=1}^{3} \\
\quad \cdot\left|\left(\Delta F+\Delta G G_{0}^{-1}\left(-Y \Theta+\dot{x}_{d}-K e\right)\right)_{i}\right|\left|(P e)_{i}\right| \leq 0 .
\end{aligned}
$$

Summarily, using the Riccati-like equation (11) the derivative $\dot{V}$ can be bounded as

$$
\begin{aligned}
\dot{V} \leq & \frac{1}{2} e^{T}\left(-K^{T} P-P K+P\right. \\
& \left.\cdot\left(\frac{1}{\rho^{2}} I-R^{-1}\left(I+\Delta G G_{0}^{-1}\right)^{T}\right) P\right) e+\frac{1}{2} \rho^{2} d^{T} d \\
\leq & \frac{1}{2} e^{T}\left(-K^{T} P-P K+P\right. \\
& \left.\cdot\left(\frac{1}{\rho^{2}} I-(1-\kappa) R^{-1}\right) P\right) e+\frac{1}{2} \rho^{2} d^{T} d \\
\leq & -\frac{1}{2} e^{T} Q e+\frac{1}{2} \rho^{2} d^{T} d .
\end{aligned}
$$

Integrating the above inequality from $t=0$ to $t=T$ yields

$$
2 V(T)+\int_{0}^{T}\|e(t)\|_{Q}^{2} d t \leq 2 V(0)+\rho^{2} \int_{0}^{T}\|d(t)\|^{2} d t
$$

for all $0 \leq T<\infty$, i.e., the $H^{\infty}$ performance in (16) is achieved [1]. Moreover, from $V(t)$ in (18) and A3) it is clear that $e(t) \in \Omega_{e} \triangleq\left\{e \mid e^{T} P e \leq 2 V(0)+\rho^{2} M_{d}\right\}$ and so $x(t) \in U_{x} \triangleq\left\{x \mid e(t) \in \Omega_{e}, x_{d}(t) \in \Omega_{d}\right\} \forall t \geq 0$. This implies all the variables are bounded.

Remark 1: The proposed controller (12) consists of three parts: the adaptive fuzzy system, $\hat{F}(x, \Theta)=Y(x) \Theta$ equipped with (15), which is used to learn the unknown dynamics $F(x)$, the VSS controller, $u_{s}$, to eliminate the effect of the approximation error, and the robust $H^{\infty}$ controller, $u_{h}$, to achieve the desired $H^{\infty}$ tracking performance. Hence, in practice this controller is a hybrid adaptive-robust controller.

Remark 2: i) Result in Theorem 1 indicates that the control design relies only on the solution of an algebraic Riccati-like equation (11). By setting $R^{-1}=\left(1 /(1-\kappa) \rho^{2}\right) I_{3 \times 3}$ and $Q$ be a diagonal matrix, a simple solution is obtained as $P=(1 / 2) K^{-1} Q$. Since a small perturbation $\Delta G(t)$ implies the bounded value $\kappa$ is smaller, there is a trade-off between the magnitude of $\Delta G(t)$ and the control gain in $u_{h}$. ii) Since the value $\epsilon_{f}$ may be made arbitrarily small by increasing the number of fuzzy If-Then rules, from (14) there is a trade-off between the number of fuzzy rules as well as the control gain in $u_{s}$.

Remark 3: From (15) it is shown that $b_{i j}-\delta \leq \theta_{i j} \leq c_{i j}+\delta$ for $1 \leq$ $j \leq m_{i}$ and $1 \leq i \leq 3$. So, it can be concluded that $\underline{b}_{i} \leq \hat{f}_{i}\left(x, \Theta_{i}\right) \leq$ $\bar{c}_{i}$ where $\underline{b}_{i} \triangleq \min _{1 \leq j \leq m_{i}}\left(b_{i j}-\delta\right)$ and $\bar{c}_{i} \triangleq \max _{1 \leq j \leq m_{i}}\left(c_{i j}+\delta\right)$. Actually, since each $\zeta_{i j}(x) \in(0,1]$ and $\sum_{j=1}^{m_{i}} \zeta_{i j}(x)=1$, we get $\underline{b}_{i} \leq \sum_{j=1}^{m_{i}}\left(b_{i j}-\delta\right) \zeta_{i j} \leq \hat{f}_{i}\left(x, \Theta_{i}\right) \leq \sum_{j=1}^{m_{i}}\left(c_{i j}+\delta\right) \zeta_{i j} \leq \bar{c}_{i}$. Based on this property if the upper and lower bounds of $f_{i}(x)$ (e.g., $\left.f_{i} \leq f_{i}(x) \leq \bar{f}_{i}\right)$ are well-known, then we can simply set $b_{i j}=$ $\bar{f}_{i}, c_{i j}=\bar{f}_{i}$ for all $j=1, \ldots, m_{i}$.

Since the nonsmoothness of $\operatorname{sgn}(\mathrm{Pe})$ results in the VSS control algorithm (14) with discontinuities between the sliding surfaces, the VSS adaptive fuzzy-based controller developed above may lead to control chattering and could excite high-frequency unmodeled modes [8]. To avoid this discontinuity we use the exponential modification to replace $u_{s}$ in (14).
Theorem 2: Consider the nonlinear chaotic Chua's circuit (1)-(3). Under the same conditions as in Theorem 1, if the VSS type control $u_{s}$ in (14) is modified to the continuous control

$$
u_{s}=-\frac{M_{E}(x)}{1-\kappa} \frac{M_{E}(x) P e}{\left\|M_{E}(x) P e\right\|+\epsilon e^{-\nu t}}
$$

where $M_{E}(x)=\sqrt{3} \epsilon_{f}+\kappa M_{e}(x)$ and $\epsilon>0, \nu>0$ are positive constants, then i) if $d \in L_{2}[0, \infty)$, an $H^{\infty}$ tracking performance is achieved; ii) if $d \in L_{\infty}[0, \infty)$, the tracking error is uniformly ultimately bounded; iii) if $d \in L_{2}[0, \infty) \cap L_{\infty}[0, \infty)$, then $\lim _{t \rightarrow \infty} e(t)=0$; and iv) all the variables are bounded.

Proof: Following the proof in Theorem 1 and using (11)-(13), (15) it can be shown that

$$
\begin{aligned}
\dot{V} \leq & -\frac{1}{2} e^{T} Q e+\frac{1}{2} \rho^{2} d^{T} d+u_{s}^{T}\left(I+\Delta G G_{0}^{-1}\right)^{T} P e \\
& +\left(\Delta F+\Delta G G_{0}^{-1}\left(-Y \Theta+\dot{x}_{d}-K e\right)\right)^{T} P e .
\end{aligned}
$$

By $u_{s}$ in (23) and the assumptions A1)-A2) we get

$$
\begin{aligned}
u_{s}^{T}(I & \left.+\Delta G G_{0}^{-1}\right)^{T} P e \\
& +\left(\Delta F+\Delta G G_{0}^{-1}\left(-Y \Theta+\dot{x}_{d}-K e\right)\right)^{T} P e \\
\leq & -\frac{\left(M_{E} P e\right)^{T}\left(I+\Delta G G_{0}^{-1}\right)^{T}\left(M_{E} P e\right)}{(1-\kappa)\left(\left\|M_{E} P e\right\|+\epsilon e^{-\nu t}\right)}+\left\|M_{E} P e\right\| \\
\leq & \epsilon e^{-\nu t} .
\end{aligned}
$$

Consequently, the derivative $\dot{V}$ can be bounded as

$$
\dot{V} \leq-\frac{1}{2} e^{T} Q e+\frac{1}{2} \rho^{2} d^{T} d+\epsilon e^{-\nu t} .
$$

Integrating the above inequality yields

$$
\int_{0}^{T}\|e(t)\|_{Q}^{2} d t \leq 2 V(0)+\frac{2 \epsilon}{\nu}\left(1-e^{-\nu T}\right)+\rho^{2} \int_{0}^{T}\|d(t)\|^{2} d t .
$$

This implies an $H^{\infty}$ tracking performance is achieved.

Next, suppose $d$ is bounded, i.e., there is an $\epsilon_{d}>0$ such that $\|d\| \leq$ $\epsilon_{d}$. The inequality (26) can be rewritten as $\dot{V} \leq-(1 / 2) \lambda_{q}\|e\|^{2}+$ $(1 / 2) \rho^{2} \epsilon_{d}^{2}+\epsilon$ where $\lambda_{q}$ denotes the minimum eigenvalue of $Q$. Then, for any given $\eta>0$ there is a choice of $\lambda_{q}$ such that $\dot{V} \leq-\xi\|e\|^{2}<0$ $\forall\|e(t)\|>\eta$ for some $\xi>0$. This implies that there is a $T>0$ such that $\|e(t)\| \leq \eta$ for all $t \geq T$ [10].

Moreover, if $d(\cdot) \in L_{2} \cap L_{\infty}[0, \infty)$, from the closed-loop error system it is clear that $\dot{e}(t)$ is uniformly bounded. This implies that $e(t)$ is uniformly continuous. Based on the Barbalat's lemma [10] and the inequality (26), it can be concluded that $\lim _{t \rightarrow \infty} e(t)=0$.

Finally, as in the proof of Theorem 1 we can conclude that all the states and signals of the closed-loop system are bounded.

Remark 4: The term $(2 \epsilon / \nu)\left(1-e^{-\nu T}\right)$ in (27) is yielded owing to the smooth modification of $u_{s}$ and can be viewed as an external disturbance. The smaller the value $\epsilon$ and the larger the value $\nu$, the less smooth is the robust controller $u_{s}$ and the smaller is the term $(2 \epsilon / \nu)\left(1-e^{-\nu T}\right)$. As $\epsilon=0$, this term is equal to zero and this $H^{\infty}$ tracking performance is reduced to (16).

In the above analyses, the input gain matrix $G(t, x)$ is assumed to be expressed as a nominal part plus a small perturbation. However, in practical applications $G(t, x)$ may be completely unknown and cannot be available directly in the control design. Without loss of generality, assume the values of $C_{1}, C_{2}$ and $L$ are nonlinear timevarying, dependent on the state variable, and unknown. For simplicity of notation, let $g_{1}(t, x) \triangleq 1 / C_{1}(t, x), g_{2}(t, x) \triangleq 1 / C_{2}(t, x)$ and $g_{3}(t, x) \triangleq 1 / L(t, x)$. 


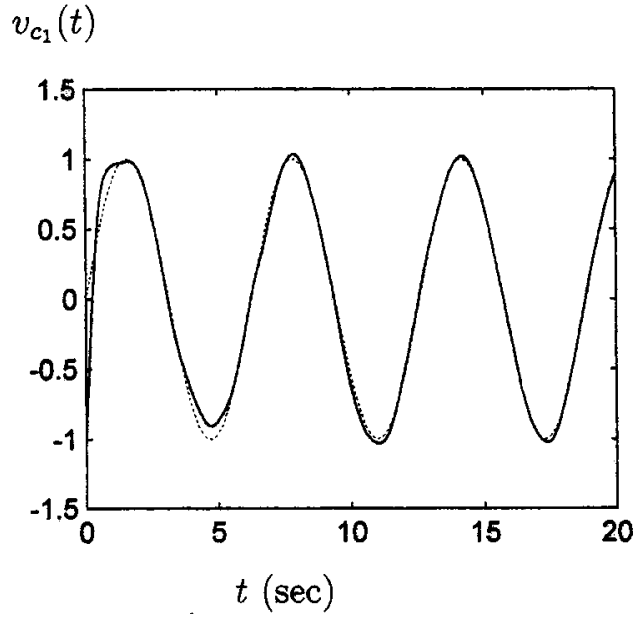

(a)

$i_{L}(t)$

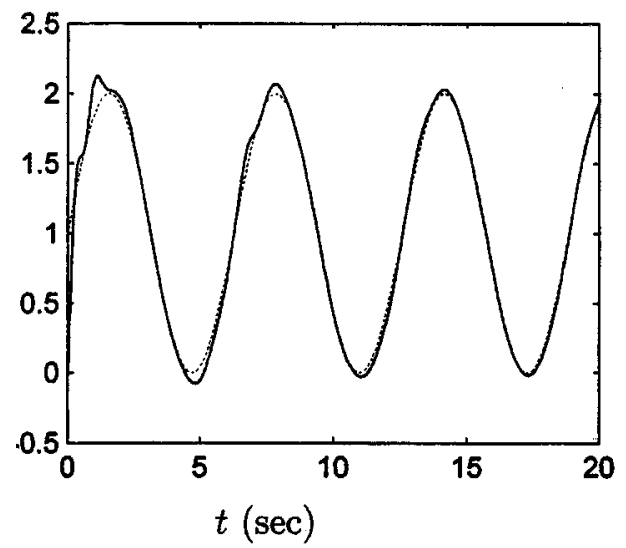

(c) $v_{c_{2}}(t)$

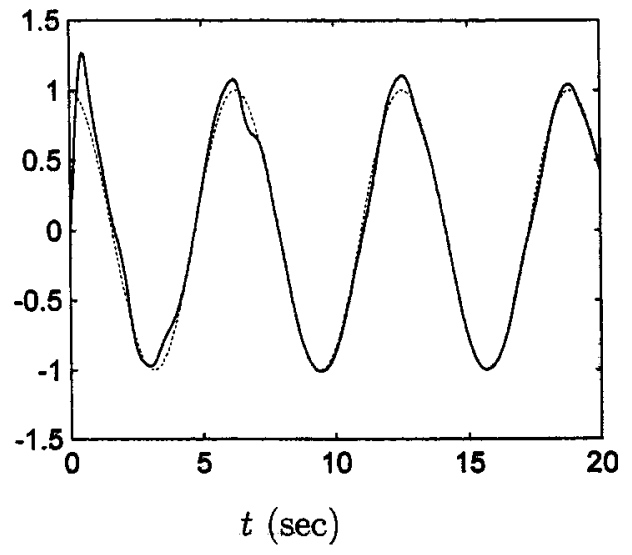

(b)

$u_{1}(t)$

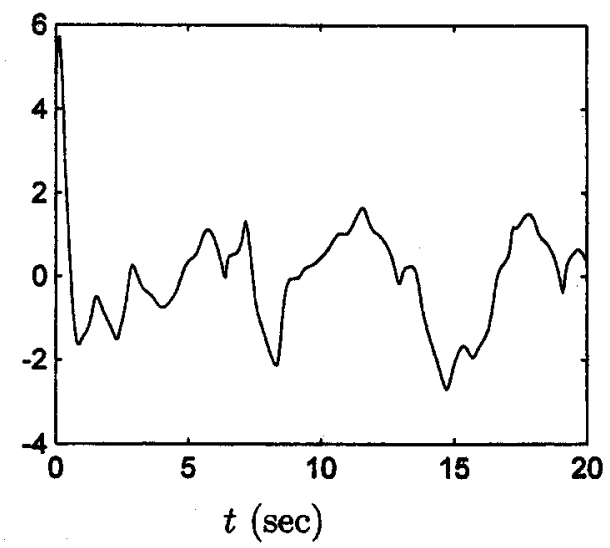

(d)

Fig. 3. The responses for the nonlinear chaotic Chua's circuit using the developed controller. (a) Capacitor voltage $v_{c_{1}}(t)$. (b) Capacitor voltage $v_{c_{2}}(t)$. (c) Inductor current $i_{L}(t)$. (d) Control input $u_{1}(t)$.

Take the fuzzy system $\hat{g}_{i}\left(x, \Theta_{g i}\right)$ to approximate the uncertain term $g_{i}(t, x)$. From $(6) \hat{g}_{i}\left(x, \Theta_{g i}\right)$ can be expressed as

$$
\hat{g}_{i}\left(x, \Theta_{g i}\right)=\zeta_{g i}^{T}(x) \Theta_{g i}, \quad i=1,2,3
$$

where $\Theta_{g i} \in R^{m_{g i}}$ for some $m_{g i}>0$ is a parameter vector and $\zeta_{g i}(x) \in R^{m_{g i}}$ is a regressive vector. Suppose the constrained regions of $\Theta_{g i}, i=1,2,3$ are convex hypercubes. That is, consider $\Omega_{g i 0} \triangleq\left\{\Theta_{g i} \mid b_{g i j} \leq \theta_{g i j} \leq c_{g i j}, 1 \leq j \leq m_{g i}\right\}$ and $\Omega_{\theta_{g i}} \triangleq\left\{\Theta_{g i} \mid b_{g i j}-\delta_{g i} \leq \theta_{g i j} \leq c_{g i j}+\delta_{g i}, 1 \leq j \leq m_{g i}\right\}$ where the values of $b_{g i j}, c_{g i j}$ and $\delta_{g i}>0$ can be arbitrarily specified. Let $\Phi_{g i} \triangleq u_{i} \zeta_{g i}(\mathrm{Pe})_{i}, i=1,2,3$ where $(\mathrm{Pe})_{i}$ denotes the $i$ element of $P e$. Therefore, the smooth projection algorithm of $\Theta_{g i}$ with respect to $\Omega_{\theta}$ is

$$
\operatorname{Proj}\left[\Phi_{g i}, \Theta_{g i}\right] \triangleq \begin{cases}\bar{\phi}_{g i j}, & \text { if }\left(\theta_{g i j}>c_{g i j} \& \phi_{g i j}>0\right) \\ \check{\phi}_{g i j}, & \text { if }\left(\theta_{g i j}<b_{g i j} \& \phi_{g i j}<0\right) \\ \phi_{g i j}, & \text { otherwise, } 1 \leq j \leq m_{g i}\end{cases}
$$

where $\bar{\phi}_{g i j} \triangleq\left(1+\left(c_{g i j}-\theta_{g i j} / \delta_{g i}\right)\right) \phi_{g i j}, \check{\phi}_{g i j} \triangleq\left(1+\left(\theta_{g i j}-\right.\right.$ $\left.\left.b_{g i j} / \delta_{g i}\right)\right) \phi_{g i j}$ and $\phi_{g i j}$ denotes the $j$ element of $\Phi_{g i}$.

Let $\Theta_{g i}^{*}, i=1,2,3$ be the optimal approximation parameters. Define the minimum approximation errors $\Delta g_{i}(t, x)=g_{i}(t, x)-$ $\hat{g}_{i}\left(x, \Theta_{g i}^{*}\right)$ and the estimated errors $\tilde{\Theta}_{g i}=\Theta_{g i}-\Theta_{g i}^{*}$. After some simple manipulations, the input matrix $G(t, x)$ is equal to

$$
G(t, x)=\hat{G}\left(x, \Theta_{g}\right)-Y_{g}(x) \tilde{\Theta}_{g}+\Delta G(t, x)
$$

where

$$
\begin{aligned}
\hat{G}\left(x, \Theta_{g}\right) & =\operatorname{diag}\left[\hat{g}_{1}\left(x, \Theta_{g 1}\right), \hat{g}_{2}\left(x, \Theta_{g 2}\right), \hat{g}_{3}\left(x, \Theta_{g 3}\right)\right] \\
Y_{g}(x) & =\operatorname{diag}\left[\zeta_{g 1}^{T}(x), \zeta_{g 2}^{T}(x), \zeta_{g 3}^{T}(x)\right] \\
\tilde{\Theta}_{g} & =\operatorname{diag}\left[\tilde{\Theta}_{g 1}, \tilde{\Theta}_{g 2}, \tilde{\Theta}_{g 3}\right] \\
\Delta G(t, x) & =\operatorname{diag}\left[\Delta g_{1}(t, x), \Delta g_{2}(t, x), \Delta g_{3}(t, x)\right] .
\end{aligned}
$$

In addition, we make the following assumption.

A4) There is a constant $0<\kappa_{g}<1$ such that $\left|\Delta g_{i}(t, x) \hat{g}_{i}^{-1}\left(x, \Theta_{g i}\right)\right| \leq \kappa_{g}, \forall 1 \leq i \leq 3$.

Theorem 3: Consider the uncertain nonlinear chaotic Chua's circuit (1)-(3). Suppose A2)-A4) are satisfied. If there exists a symmetric positive matrix $P=P^{T}>0$ satisfying the Riccati-like equation

$$
-K^{T} P-P K+Q+P\left(\frac{1}{\rho^{2}} I_{3 \times 3}-\left(1-\kappa_{g}\right) R^{-1}\right) P=0
$$

then the following VSS adaptive fuzzy-based control law

$$
u=\hat{G}^{-1}\left(x, \Theta_{g}\right)\left(-Y(x) \Theta+\dot{x}_{d}-K e+u_{h}+u_{s}\right)
$$




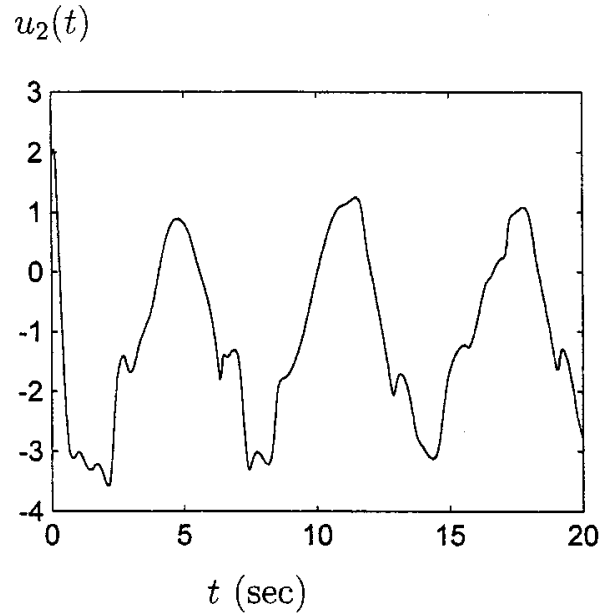

(e)

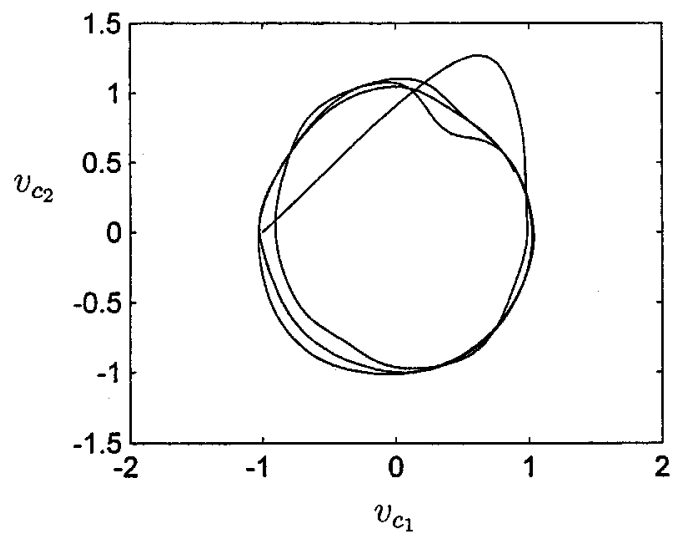

(g) $u_{3}(t)$

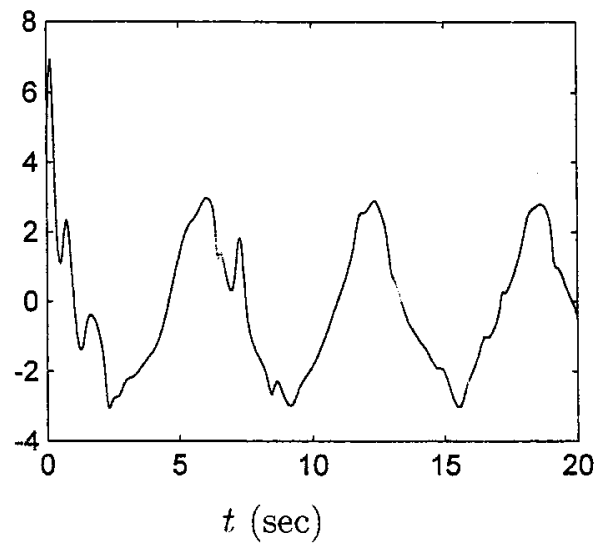

(f)

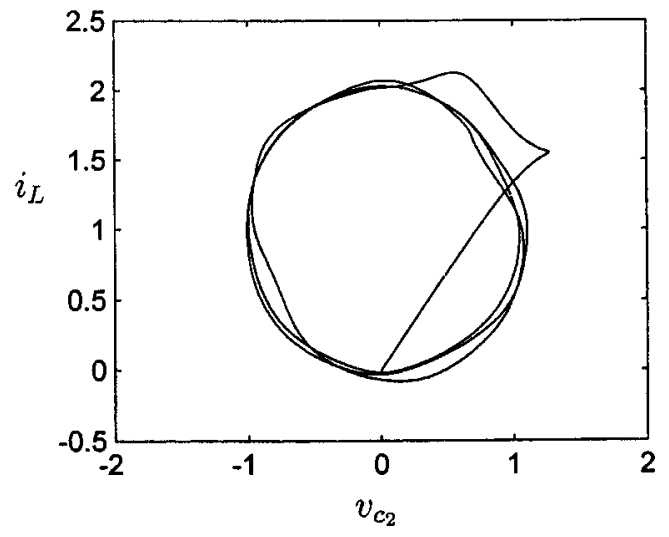

(h)

Fig. 3. (Continued) The responses for the nonlinear chaotic Chua's circuit using the developed controller. (e) Control input $u_{2}(t)$. (f) Control input $u_{3}(t)$. (g) phase-plane trajectory $v_{c_{1}}-v_{c_{2}}$. (h) Phase-plane trajectory $v_{c_{2}}-i_{L}$.

with $u_{h}$ in (13), $u_{s}=-\left(\epsilon_{f}+\kappa_{g} M_{e}\right) \operatorname{sgn}(P e) /\left(1-\kappa_{g}\right), \dot{\Theta}$ in (15), and $\dot{\Theta}_{g i}=\gamma_{g i} \operatorname{Proj}\left[\Phi_{g i}, \Theta_{g i}\right]$ in (29) with $b_{g i j}-\delta_{g i}>0 \forall 1 \leq j \leq$ $m_{g i}, 1 \leq i \leq 3$ where $\gamma_{g i}>0$ are the adaptive gains, guarantees all the variables of the closed-loop system are bounded and an $H^{\infty}$ tracking performance is achieved.

Proof: From (30) and (32), the error dynamic equation (4) can be rewritten as

$$
\begin{aligned}
\dot{e}= & -K e-Y(x) \tilde{\Theta}+\Delta F(x)+\Delta G(t, x) u \\
& -Y_{g}(x) \tilde{\Theta}_{g} u+u_{h}+u_{s}+d .
\end{aligned}
$$

Choose a Lyapunov function as

$$
W=\frac{1}{2} e^{T} P e+\frac{1}{2 \gamma} \tilde{\Theta}^{T} \tilde{\Theta}+\sum_{i=1}^{3} \frac{1}{2 \gamma_{g i}} \tilde{\Theta}_{g i}^{T} \tilde{\Theta}_{g i}
$$

As in the proof of Theorem 1, the derivative $\dot{W}$ can be bounded as

$$
\begin{aligned}
\dot{W} \leq & -\frac{1}{2} e^{T} Q e+\frac{1}{2} \rho^{2} d^{T} d \\
& -\sum_{i=1}^{3} u_{i} \tilde{\Theta}_{g i}^{T} \zeta_{g i}(P e)_{i}+\sum_{i=1}^{3} \frac{1}{\gamma_{g i}} \dot{\Theta}_{g i}^{T} \tilde{\Theta}_{g i} .
\end{aligned}
$$

It can be shown that $\left(1 / \gamma_{g i}\right) \dot{\Theta}_{g i}^{T} \tilde{\Theta}_{g i}-u_{i} \tilde{\Theta}_{g i}^{T} \zeta_{g i}(P e)_{i} \leq 0$ and $\Theta_{g i}(t) \in \Omega_{\theta_{g i}} \forall t \geq 0$. Similarly to the proof of Theorem 1 , an $H^{\infty}$ performance is achieved and all the variables are bounded.
Finally, we shall show that the matrix $\hat{G}\left(x, \Theta_{g}\right)$ is invertible. Let $\underline{b}_{g i} \triangleq \min _{1 \leq j \leq m_{g i}}\left(b_{g i j}-\delta_{g i}\right)$ and $\bar{c}_{g i} \triangleq \max _{1 \leq j \leq m_{g i}}\left(c_{g i j}+\delta_{g i}\right)$. As in Remark 3, it can be concluded that $\underline{b}_{g i} \leq \hat{g}_{i}\left(x, \Theta_{g i}\right) \leq \bar{c}_{g i}$, $i=1,2,3$. Then, if we suitably choose $b_{g i j}$ and $\delta_{g i}$ such that $\underline{b}_{g i}>0$, $\hat{G}\left(x, \Theta_{g}\right)$ is invertible and so the controller is well-defined.

\section{Simulation EXAMPLE}

Consider the tracking control problem of the uncertain chaotic Chua's circuit shown in Fig. 1. For the convenience of simulation, the nominal parameters are given as $C_{10}=1, C_{20}=0.5$ and $L_{0}=1$, and the perturbations are given as $\Delta C_{1}=0.1+0.1 \cos (t / 2), \Delta C_{2}=0.1$ and $\Delta L=0.15$. The perturbed resistors are characterized by $R=5+\sin (t / 2)$ and $g\left(v_{c_{1}}\right)=-v_{c_{1}}+0.02 v_{c_{1}}^{3}$. The exogenous disturbances are $d_{1}=0.25 \sin (2 t) \exp (-0.1 t)$, $d_{2}=0.2 \cos (2 t) \exp (-0.1 t)$ and $d_{3}=0.2 \sin (2 t) \exp (-0.1 t)$.

Let the desired voltage and current be $x_{d_{1}}(t)=\sin (t), x_{d_{2}}(t)=$ $\cos (t)$ and $x_{d_{3}}(t)=1+\sin (t)$. The VSS adaptive fuzzy-based $H^{\infty}$ tracking control law developed in Theorem 2 is employed to treat this trajectory planning problem. We divide the design into three steps.

Step 1: Let $x_{1}=v_{c_{1}}, x_{2}=v_{c_{2}}, x_{3}=i_{L}$ and $x_{4}=v_{c_{1}}-v_{c_{2}}$. Define five fuzzy sets for each $x_{i}, i=1,2,3,4$ with labels $F_{i}^{1}$ (negative large), $F_{i}^{2}$ (near -0.5), $F_{i}^{3}$ (near 0), $F_{i}^{4}$ (near 0.5), and $F_{i}^{5}$ (positive large) which are characterized by the membership functions, respectively, $\mu_{F_{i}^{1}}\left(x_{i}\right)=1 /\left(1+\exp \left(5\left(x_{i}+1\right)\right)\right), \mu_{F_{i}^{2}}\left(x_{i}\right)=\exp \left(-2\left(x_{i}+\right.\right.$ $\left.0.5)^{2}\right), \mu_{F_{i}^{3}}\left(x_{i}\right)=\exp \left(-2 x_{i}^{2}\right), \mu_{F_{i}^{4}}\left(x_{i}\right)=\exp \left(-2\left(x_{i}-0.5\right)^{2}\right)$, and $\mu_{F_{i}^{5}}\left(x_{i}\right)=1 /\left(1+\exp \left(-5\left(x_{i}-1\right)\right)\right)$. 
Now, construct the fuzzy approximator $\hat{f}_{1}\left(x, \Theta_{1}\right)$ in (7). 25 fuzzy rules are included

$$
\begin{aligned}
& R_{i j}^{(l)}: \text { If } x_{1} \text { is } F_{1}^{i} \text { and } x_{4} \text { is } F_{4}^{j} \text {, Then } y \text { is } F_{i j}^{l} \text {, } \\
& \quad \text { for } i, j=1, \ldots, 5 \quad l=1, \ldots, 25 \text {. }
\end{aligned}
$$

The fuzzy regressive vector in (7) is chosen to be $\zeta_{1}=\left[\zeta_{11}, \zeta_{12}\right.$, $\left.\ldots, \zeta_{125}\right]^{T} \in R^{25}$ with components as $\zeta_{11}(x)=\mu_{F_{1}^{1}}\left(x_{1}\right) \mu_{F_{4}^{1}}\left(x_{4}\right) /$ $D_{1}, \zeta_{12}(x)=\mu_{F_{1}^{1}}\left(x_{1}\right) \mu_{F_{4}^{2}}\left(x_{4}\right) /, \ldots, \zeta_{124}(x)=\mu_{F_{1}^{5}}\left(x_{1}\right) \mu_{F_{4}^{4}}$ $\left(x_{4}\right) / D_{1}$, and $\zeta_{125}(x)=\mu_{F_{1}^{5}}\left(x_{1}\right) \mu_{F_{4}^{5}}\left(x_{4}\right) / D_{1}$ where $D_{1} \stackrel{4}{=}$ $\sum_{i=1}^{5} \sum_{j=1}^{5} \mu_{F_{1}^{i}}\left(x_{1}\right) \mu_{F_{4}^{j}}\left(x_{4}\right)$. Hence, $f_{1}(x)$ is approximated by $\hat{f}_{1}\left(x, \Theta_{1}\right)=\zeta_{1}^{T}(x) \Theta_{1}$ with $\Theta_{1} \triangleq\left[\theta_{11}, \theta_{12}, \ldots, \theta_{125}\right]^{T} \in R^{25}$ Next, 25 fuzzy rules are included in $\hat{f}_{2}\left(x, \Theta_{2}\right)$ :

$$
\begin{array}{r}
R_{i j}^{(l)}: \text { If } x_{3} \text { is } F_{3}^{i} \text { and } x_{4} \text { is } F_{4}^{j} \text {, Then } y \text { is } F_{i j}^{l}, \\
\quad \text { for } i, j=1, \ldots, 5 \quad l=26, \ldots, 50 .
\end{array}
$$

Choose $\zeta_{2}=\left[\zeta_{21}, \zeta_{22}, \ldots, \zeta_{225}\right]^{T} \in R^{25}$ with $\zeta_{21}(x)=\mu_{F_{3}^{1}}\left(x_{1}\right)$ $\mu_{F_{4}^{1}}\left(x_{4}\right) / D_{2}, \zeta_{22}(x)=\mu_{F_{3}^{1}}\left(x_{1}\right) \times \mu_{F_{4}^{2}}\left(x_{4}\right) / D_{2}, \ldots, \zeta_{225}(x)=$ $\mu_{F_{3}^{5}}\left(x_{1}\right) \mu_{F_{4}^{5}}\left(x_{4}\right) / D_{2}$, and $\stackrel{3}{D}{ }_{2}=\sum_{i=1}^{5} \sum_{j=1}^{5} \mu_{F_{3}^{i}}\left(x_{1}\right) \mu_{F_{4}^{j}}\left(x_{4}\right)$. Hence, $\hat{f}_{2}\left(x, \Theta_{2}\right)=\zeta_{2}^{T}(x) \Theta_{2}$ with $\Theta_{2} \triangleq\left[\theta_{21}, \theta_{22}, \ldots, \theta_{225}\right]^{T} \in$ $R^{25}$. Finally, 5 fuzzy rules are included in $\hat{f}_{3}\left(x, \Theta_{3}\right)$ :

$$
\begin{aligned}
& R_{i}^{(l)}: \text { If } x_{2} \text { is } F_{2}^{i} \text {, Then } y \text { is } F_{i}^{l} \\
& \quad \text { for } i=1, \ldots, 5, l=51, \ldots, 55 \text {. }
\end{aligned}
$$

Choose $\zeta_{3}=\left[\zeta_{31}, \ldots, \zeta_{35}\right]^{T} \in R^{5}$ with $\zeta_{31}(x)=\mu_{F_{2}^{1}}\left(x_{2}\right) /$ $D_{3}, \ldots, \zeta_{35}(x)=\mu_{F_{2}^{5}}\left(x_{2}\right) / D_{3}$, and $D_{3}=\sum_{i=1}^{5} \mu_{F_{2}^{i}}\left(x_{2}\right)$. Hence, $\hat{f}_{3}\left(x, \Theta_{3}\right)=\zeta_{3}^{T}(x) \Theta_{3}$ with $\Theta_{3} \triangleq\left[\theta_{31}, \ldots, \theta_{35}\right]^{T} \in R^{5}$. Consequently, $F(x)$ is approximated by $\hat{F}(x, \Theta)=Y(x) \Theta$ with $Y(x) \triangleq \operatorname{diag}\left[\zeta_{1}^{T}(x), \zeta_{2}^{T}(x), \zeta_{3}^{T}(x)\right]$ and $\Theta \triangleq\left[\Theta_{1}^{T}, \Theta_{2}^{T}, \Theta_{3}^{T}\right]^{T}$.

Step 2: Select $K=2 I_{3 \times 3}, \rho=0.5$ and $Q=2 I_{3 \times 3}$. Set $\kappa=0.2$. Consequently, take $R=(1-\kappa) \rho^{2} I_{3 \times 3}=0.2 I_{3 \times 3}$ and solving the equation in (11) yields $P=(1 / 2) K^{-1} Q=0.5 I_{3 \times 3}$.

Step 3: Obtain the tracking controller (12), (13), (15), (23) with $\epsilon_{f}=0.2, \epsilon=1, \nu=0.1, \gamma=200, b_{i j}=-5, c_{i j}=5 \forall i j$, and $\delta=0.5$.

Choose the initial conditions $v_{c_{1}}(0)=-1, v_{c_{2}}(0)=0, i_{L}(0)=0$, and $\Theta(0)=0_{55 \times 1}$. The simulation results are shown in Fig. 3. The phase-plane trajectory $v_{c_{1}}-v_{c_{2}}$ converges to a circle of unit radius centered at $(0,0)$ and $v_{c_{2}}-i_{L}$ converges to a circle of unit radius centered at $(0,1)$. These simulation results indicate that the tracking performance is nice and consequently the effects due to parametric uncertainties and external disturbances in chaotic Chua's control circuits can be efficiently diminished by the developed control algorithm.

\section{Conclusions}

An adaptive fuzzy-based tracking control design incorporating with a standard VSS control algorithm and a nonlinear $H^{\infty}$ control algorithm has been proposed and solved for uncertain nonlinear chaotic Chua's circuits. The hybrid adaptive-robust tracking controller developed in this study guarantees that all the states and signals of the closed-loop system are bounded and an $H^{\infty}$ tracking control is achieved. Compared with the previous investigations which also address the problem of controlling Chua's circuits this paper can be extended to handle a broader class of nonlinear chaotic Chua's circuits in the presence of plant uncertainties and external disturbances, and so this design is quite useful from the viewpoint of practical applications. Finally, a simulation example is included to confirm the validity and performance of the developed control scheme.

\section{REFERENCES}

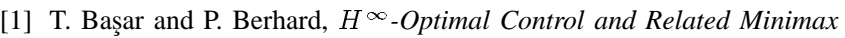
Problems. Berlin, Germany: Birkhäuser, 1990.

[2] L. Chen and G. Chen, "Fuzzy predictive control of uncertain chaotic systems using time series," Int. J. Bifurcation Chaos, vol. 9, no. 4, pp. 757-767, 1999.

[3] B. S. Chen, C. H. Lee, and Y. C. Chang, " $H^{\infty}$ tracking design of uncertain nonlinear SISO systems: Adaptive fuzzy approach," IEEE Trans. Fuzzy Syst., vol. 4, pp. 32-43, Feb. 1996.

[4] G. Chen and X. Dong, "On feedback control of chaotic continuous-time systems," IEEE Trans. Circuits Syst., vol. 40, pp. 591-601, Sept. 1993.

[5] G. Chen and X. Dong, From Chaos to Order-Methodologies, Perspectives, and Applications. Singapore: Word Scientific, 1998.

[6] L. O. Chua, M. Komuro, and T. Matsumoto, "The double scroll family: I and II," IEEE Trans. Circuits Syst., vol. 33, pp. 1072-1118, Nov. 1996.

[7] K. Fischle and D. Schröder, "An improved stable adaptive fuzzy control method," IEEE Trans. Fuzzy Syst., vol. 7, pp. 27-40, Feb. 1999.

[8] J. Y. Hung, W. Gao, and J. C. Hung, "Variable structure control: A survey," IEEE Trans. Ind. Electron., vol. 40, pp. 2-22, Feb. 1993.

[9] A. Isidori, Nonlinear Control Systems, 2nd ed. Berlin, Germany: Springer-Verlag, 1989.

[10] H. K. Khalil, "Adaptive output feedback control of nonlinear systems represented by input-output models," IEEE Trans. Automat. Contr., vol. 41, pp. 177-188, Feb. 1996.

[11] M. J. Ogorzalek, "Taming chaos-Part II: Control," IEEE Trans. Circuits Syst., vol. 40, pp. 700-706, Oct. 1993.

[12] R. Ordóñez, J. Zumberge, J. T. Spooner, and K. M. Passino, "Adaptive fuzzy control: Experiments and comparative analyzes," IEEE Trans. Fuzzy Syst., vol. 5, pp. 167-188, May 1997.

[13] J. T. Spooner and K. M. Passino, "Stable adaptive control using fuzzy systems and neural networks," IEEE Trans. Fuzzy Syst., vol. 4, pp. 339-359, Aug. 1996.

[14] M. Storace, M. Parodi, and D. Robatto, "A Hysteresis-based chaotic circuit: Dynamics and applications," Int. J. of Circuit Theory Appl., vol. 27, pp. 527-542, 1999.

[15] K. Tanaka, T. Ikeda, and H. O. Wang, "A unified approach to controlling Chaos via an LMI-based fuzzy control system design," IEEE Trans. Circuits Syst. I, vol. 45, pp. 1021-1040, Oct. 1998.

[16] L. X. Wang, A Course in Fuzzy Systems and Control. Englewood Cliffs, NJ: Prentice-Hall, 1997.

[17] H. O. Wang and E. H. Abed, "Bifuraction control of a chaotic system," Automatica, vol. 33, no. 9, pp. 1213-1226, 1995.

[18] J. Xu, G. Chen, and L. S. Shieh, "Digital redesign for controlling the chaotic Chua's circuit," IEEE Trans. Aerosp. Electron. Syst., vol. 32, pp. 1488-1499, Oct. 1996. 